\title{
Artesanías autóctonas como atractivo turístico en la provincia de Santa Elena, Ecuador.
}

Indigenous handicrafts as tourist attraction in the province of Santa Elena, Ecuador.

Arnaldo Efrén Mendoza Tarabó. ${ }^{1}$, Clemente Hugo Ramírez Frías. ${ }^{2}$, Jhony Ernesto Yumisaca Tuquinga. ${ }^{3} \&$ Silvia Paola Peralta Mendoza. ${ }^{4}$

\section{Resumen.}

Pese a la demanda de objetos fabricados en serie con el uso de la tecnología actual las artesanías autóctonas en Ecuador han logrado mantenerse vigentes, debido a la habilidad de sus artesanos y a los saberes ancestrales que aplican en ellas, La investigación buscó demostrar el potencial de la artesanía como un atractivo en la zona de estudio de la provincia Santa Elena. Se lleva a efecto una metodología bibliográfica y empírica con la aplicación de encuestas a los principales involucrados en este sector. Los resultados muestran la importancia, motivaciones, distribución del gasto por compra que tienen las personas, así como el alcance de la publicidad de las artesanías. Los visitantes solicitan constantemente los productos elaborados con paja toquilla y marfil vegetal (tagua), representativos de esta zona costera, por su autenticidad, pese a la escasa información proporcionada por los entes encargados.

Palabras claves: Artesanías autóctonas, atractivo turístico, identidad, saberes ancestrales.

\section{Abstract.}

Native handicrafts from the province of Santa Elena in Ecuador have managed to remain in force despite the demand for objects manufactured in series with the use of technology, due to the skill of its artisans and the ancestral knowledge they apply to them. The

\footnotetext{
${ }^{1}$ Universidad Estatal Península de Santa Elena, La Libertad, Ecuador. emendoza@upse.edu.ec

${ }^{2}$ Universidad de La Habana, Cuba. clemenhugo_ramirez@ftur.uh.cu

${ }^{3}$ Universidad Estatal Península de Santa Elena, La Libertad, Ecuador. jyumisaca@ upse.edu.ec

${ }^{4}$ Universidad Estatal Península de Santa Elena, La Libertad, Ecuador. speralta@upse.edu.ec
} 
research sought to demonstrate the potential of craftsmanship as an attraction in the study area, carrying out a bibliographic and empirical methodology with the application of surveys to the main stakeholders in this sector. The results show the importance, motivations, distribution of spending per purchase that people have, as well as the scope of advertising of handicrafts. Visitors constantly request products made with toquilla straw and vegetable ivory (tagua), representative of this coastal area, for its authenticity, there being little information provided by the entities in charge.

Keywords: Native handicrafts, tourist attraction, identity, ancestral knowledge.

\section{Introducción.}

La Organización Mundial del Turismo (OMT) en su Panorama OMT del turismo internacional (OMT, 2017:2), anuncia que el turismo "se ha distinguido por un crecimiento prácticamente ininterrumpido a lo largo del tiempo, a pesar de crisis ocasionales, demostrando siempre su fortaleza y su resistencia" [1].

Esta actividad, que repercute en los ámbitos sociales, económicos y ambientales, ha sido priorizada dentro de la matriz productiva del Ecuador, que comprende, entre otros aspectos, estimular emprendimientos turísticos en comunidades que podrían dinamizar la económica de las comunidades y de esa manera satisfacer las exigencias y necesidades que se plantean, como lo muestra el Plan Nacional del Buen Vivir (PNBV) 2017-2021 (2017:77) al especificar varias actividades que se vinculan a la generación "de trabajo como las artesanías, vestimenta, turismo, servicios de apoyo y comercio" [2].

Al destacar en las comunidades actividades como las artesanías se hace referencia a valores patrimoniales que forman parte de la cultura popular cuyo atractivo está sostenido por los saberes ancestrales, lo que a su vez, los hace frágiles ante la fuerte actividad del mercado que conlleva el turismo. En el vínculo necesario entre la cultura popular y el turismo se cometen no pocos errores en la actividad de gestión, sobre todo si se trata de tradiciones autóctonas como algunas producciones artesanales. El más común es el de construir una falsa cultura que distorsiona los valores esenciales de la cultura auténtica local. Sin embargo muchas veces la presencia turística permite que sobrevivan expresiones artísticas tradicionales casi olvidadas, se fomenta el desarrollo de artes manuales y se establecen nuevos mercados para artesanos, donde coexisten lo auténtico y autóctono con las meras imitaciones baratas.

Estas cuestiones planteadas en la relación entre el turismo y las artesanías inciden sobre áreas importantes de la identidad cultural, de ahí la importancia que tiene el abordaje científico de su puesta en valor turístico. Su realización posibilita crear condiciones para un diseño estratégico que permita el desarrollo sostenible del turismo cultural con referente en las artesanías autóctonas. Para esta investigación se considera artesanía autóctona aquella que conserva la tradición ancestral al trabajar con elementos característicos y representativos que han sido transferidos de generación en generación, se refleja en expresiones artísticas plasmadas en objetos confeccionados de manera manual con técnicas artesanales tradicionales que forman parte del patrimonio de los pueblos así como de su identidad. 
Las artesanías autóctonas han enfrentado, a partir de la revolución industrial, significativos impactos que han amenazado su subsistencia, no obstante han logrado mantenerse a salvo gracias a sus características únicas e irrepetibles que le dan un valor diferenciado ante aquellas elaboradas en serie, las que impactan en la actividad de artesanos de las áreas rurales. El artesano, de acuerdo a Silva (2005), plasma en los objetos elaborados la autenticidad de la artesanía como un valor moral, permitiendo que quienes obtengan el producto final estén seguros de que es genuino [3].

El contexto social y geográfico en que se desarrolla el estudio es en Santa Elena, una de las provincias costeras del Ecuador que presenta, además del turismo de sol y playa, una amplia oferta artesanal que involucra productos elaborados en las comunidades que trabajan con materias primas naturales como paja toquilla, tagua, madera, concha, caña guadua, entre otras. Una de las regiones protagonistas en esta actividad en la provincia es Manglaralto, parroquia que cuenta con varias comunidades que se dedican al trabajo de artesanías autóctonas.

Los objetivos de este estudio buscan determinar, ante la problemática de sus contradicciones y factores negativos, el potencial de la artesanía autóctona local como producción cultural susceptible de ser promovida como atractivo turístico capaz de generar ingresos en la región de Santa Elena, convirtiéndola en una alternativa de fuente de empleo en las comunidades rurales como el caso de Manglaralto, pero a la vez fortaleciendo la identidad y la conservación de sus recursos culturales y naturales.

La investigación que se presenta aborda el análisis de trabajos relacionados con el sector artesanal a través del método de revisión bibliográfica, además de la aplicación de encuestas a los principales involucrados en la temática. El artículo aporta, con una visión objetiva, resultados sobre la producción artesanal autóctona presente en el lugar de estudio, así como también las principales características y preferencias de sus consumidores, una información con la cual no se contaba hasta esta exploración inicial actual.

\section{Métodos}

Se emplearon una diversidad de métodos que correspondieron a su aplicación de acuerdo a la complejidad y avance de la investigación.

1. Se empleó el análisis bibliográfico mediante la revisión de estudios relacionados con la temática de las artesanías en general, y de estas en su relación con el turismo, considerando el examen de documentos sobre la planificación en el sector turístico del Ecuador.

2. Los elementos esenciales medidos a través de la búsqueda de información, tanto de las encuestas y las entrevistas, estuvieron dirigidas a identificar las preferencias de gastos de los visitantes en la compra de artesanías, las principales materias primas naturales que se emplean en la elaboración y la relación de la problemática que se presenta en el sector artesanal. Para la confección de las encuestas se consideró el cuestionario que utiliza la UNESCO, en el documento Índice Unesco Artesanía-Turismo (2004) que refleja principalmente el gasto diario de turistas en productos artesanales [4].

Las encuestas aplicadas a los principales involucrados en la provincia de Santa Elena (empresarios turísticos, artesanos y visitantes) buscaron determinar cuáles son las principales materias prima empleadas para los productos artesanales que se ofertan. La 
variable "materia prima" utilizada en mostrar los resultados permitió simplificar la relación de productos al dividirlos por grupos en relación al material de origen. En este caso la paja toquilla corresponde a dos productos principales, sombreros y carteras; Tagua, (marfil vegetal) a llaveros, collares; arcilla a vasijas, réplicas de cultura; metal a joyería diversa; conchas a figuras de animales; piedra arenisca a réplica de culturas; papel a portarretratos y tarjetas.

3. Se aplicaron entrevistas a las principales autoridades del sector público de la provincia de Santa Elena entre ellas la representante del Ministerio de Turismo, el Director de Turismo del Gobierno Autónomo Descentralizado (GAD) Municipal de Salinas, principal balneario del Ecuador, así como también al Gerente de la Empresa Municipal de Turismo del GAD de Santa Elena, territorio donde se encuentra la mayor producción artesanal de esta provincia y a la que pertenecen las comunidades de la parroquia Manglaralto.

4. Se realizó un primer estudio exploratorio en la demanda de los productos artesanales mediante la aplicación de encuestas, con una muestra aleatoria que permitió tener indicios de las artesanías como un atractivo para los visitantes. Las encuestas fueron aplicadas a los turistas que se encontraron en las poblaciones de Libertador Bolívar, Dos Mangas y Montañita, las dos primeras comunidades que concentran la mayor producción de artesanías elaboradas con diferentes materias primas y la tercera el destino turístico más representativo en la provincia que oferta objetos artesanales. El levantamiento de datos se efectuó entre los meses de julio y agosto aprovechando la temporada de visitas de grupos de turistas nacionales además de turistas extranjeros.

5. De la misma manera se levantó información a los artesanos de las poblaciones antes mencionadas para registrar el consumo de los principales objetos que compraron los visitantes, la muestra se eligió de forma intencional escogiéndose los talleres artesanales que cuentan con mayor demanda.

6. Finalmente se realizó una entrevista estructurada a un grupo de 8 profesionales máster en turismo de la carrera de Gestión y Desarrollo Turístico de la Universidad Estatal Península de Santa Elena, que aportó a la determinación del potencial que tienen las artesanías autóctonas de la parroquia Manglaralto.

\section{Resultados}

Entre los resultados encontrados del análisis de estudios sobre las artesanía en general se puede citar a Fernández de Paz (2015) quien categoriza algunas tipificaciones artesanas, la "urbana o neoartesanía", que hace referencia a aquella que asume de manera natural los cambios intencionales en los productos realizados; de la misma forma en el lado contrario señala a la "artesanía tradicional", la cual mantiene las características culturales de los artesanos que las confeccionan [5], aspecto con el que coincide Tudorache (2016) al señalar que este patrimonio se manifiesta a través de procesos, frases, conocimientos y habilidades incluidas en los objetos que elaboran, reconocidos como parte de su cultura, precisamente a lo que se hacemos referencia como artesanía autóctona [6].

Por su parte Turok (2013) destaca que Latinoamérica cuenta con artesanos y artesanas que mantienen características propias heredadas por generaciones en la elaboración de productos con materia prima del entorno en el que se desenvuelven, además de la aplicación de técnicas ancestrales que los identifica a los cuales los denomina "artesanos por tradición”, pero dentro de ese grupo aún se presenta una subdivisión: la primera hace 
referencia a aquellos individuos de los pueblos originarios que ven en la producción artesanal una alternativa para cubrir sus necesidades básicas y la segunda que vincula a grupos extranjeros que introdujeron nuevos materiales y técnicas, durante el período de la colonización, controlando la producción de los diferentes objetos creados [7].

Swanson y Timothy (2012), examinan las investigaciones en relación a las artesanías y lo que representan para los visitantes, analizan para ello su perspectiva histórica en cuanto al desarrollo de estudios en este sentido, el simbolismo que encierran y su relación como mercancía comercializable, presentan finalmente sugerencias para el enfoque de nuevos trabajos debido a que es un área poco estudiada dentro de la investigación turística [8]. Los impactos negativos que se han producido en las microempresas artesanales, integradas por el grupo familiar en su mayoría y por las asociaciones que existen para comercializar su producto, son considerables de acuerdo a lo especificado por Hernández Girón y otros autores (2007:79). Entre los aspectos que forman parte de esta problemática podemos mencionar [9]: Abuso hacia los artesanos por parte de organizaciones que comercializan sus productos, debido al escaso o casi nulo conocimiento sobre las técnicas de mercadeo; nivel de producción bajo, poco estable, presentando limitado acceso a la materia prima; la artesanía empieza a adaptarse a las necesidades de la demanda perdiendo lo tradicional; presentan desventajas al competir con productos artesanales de tipo industrial; la tecnología poco adecuada y la falta de capital se presentan como el resultado de una baja calidad en los productos; impacta además el desconocimiento del proceso administrativo lo cual hace que muchos de ellos no lleven registros de ventas, asignando precios bajos al no tomar en cuenta la mano de obra.

Por su parte el Ministerio de Turismo del Ecuador presentó en el Plan Estratégico de Desarrollo de Turismo Sostenible para el Ecuador - PLANDETUR 2020 (2007), la Línea de Productos al Turismo Cultural que integra a Mercados y Artesanías y señala en esta modalidad a aquellas actividades y experiencias en el ámbito cultural que atraen y promueven el turismo, esto implica, entre otras cosas, adentrarse en las formas de vida de las comunidades, su entorno y los aspectos que son parte de su identidad [10].

De acuerdo a lo señalado por la ex Ministra de Industrias y Productividad, Verónica Sión de Josse (2013), la actividad artesanal involucra, directa e indirectamente, a 4,5 millones de ecuatorianos, lo que representa un $32,7 \%$ de la población económicamente activa, por ello el sector es una prioridad del Gobierno: no solo por su contribución económica, sino porque constituye un amplio segmento de personas, actores clave en la preservación material e inmaterial del patrimonio cultural del pueblo. El sector está constituido por más del 80\% de unidades básicas familiares y la generación de empleo significa un 12,3\% de la población urbana a nivel nacional [11]. El Análisis del Sector Artesanías (PROECUADOR, 2013:33) elaborado por la Dirección de Inteligencia Comercial e Inversiones del Ecuador y la Promoción de Exportaciones Especialista Sectorial de Artesanías, señala que "las oportunidades comerciales para el sector artesanal que se pueden aprovechar a nivel mundial" [12], son:

1. Las artesanías a mano puede generar un importante desarrollo a los pequeños artesanos.

2. Los materiales usados son exóticos a niveles internacionales o únicos en el mundo. 
3. Los únicos países productores de tagua a nivel mundial son Ecuador, Brasil, Venezuela, Perú, Colombia y Panamá.

4. Genera un impacto directo en la producción de productos con valor agregado, de los países.

5. Incrementa la productividad laboral y fortalece el desarrollo de la economía del país.

6. Industria de baja complejidad tecnológica, diferencia cada producto por ser único.

7. La artesanía ecuatoriana es apreciada por su originalidad y costo razonable.

El Fondo Nacional para el Fomento de las Artesanías - FONART (2015:14), se ajusta a la concepción de artesanía que es objeto de estudio en este artículo, y hace referencia a que es un "producto de identidad cultural comunitaria, hecho por procesos manuales continuos, auxiliados por implementos rudimentarios y algunos de función mecánica que aligeran ciertas tareas" [13].

En estos territorios rurales la materia prima la obtienen los artesanos generalmente en el sitio donde habitan. El saber ancestral heredado a través del uso de técnicas empleadas por generaciones permite la creación de diversos objetos que tienen además valores simbólicos característicos de la región y son el reflejo de su cultura. Esta descripción complementa la diferenciación de los productos confeccionados con un carácter de único debido a que el mismo modelo presenta diferencias por haber sido elaborados en un mayor porcentaje de manera manual.

La región costera de Santa Elena presenta, además del turismo de sol y playa, una amplia oferta artesanal, que involucra productos elaborados por las hábiles manos de hombres y mujeres en las comunidades que trabajan con paja toquilla, tagua, madera, concha, caña guadua, entre otras. Manglaralto, parroquia ubicada hacia el norte de la provincia, cuenta con 18 comunidades, de las cuales el $66,7 \%$ (12 de ellas) se dedican al trabajo de artesanías autóctonas con una variedad de materias prima que contribuyen a su diversidad en productos. Un porcentaje en este sentido se refleja en el siguiente gráfico.

Gráfico 1: Grupos de productos artesanales según materia prima en la parroquia Manglaralto.

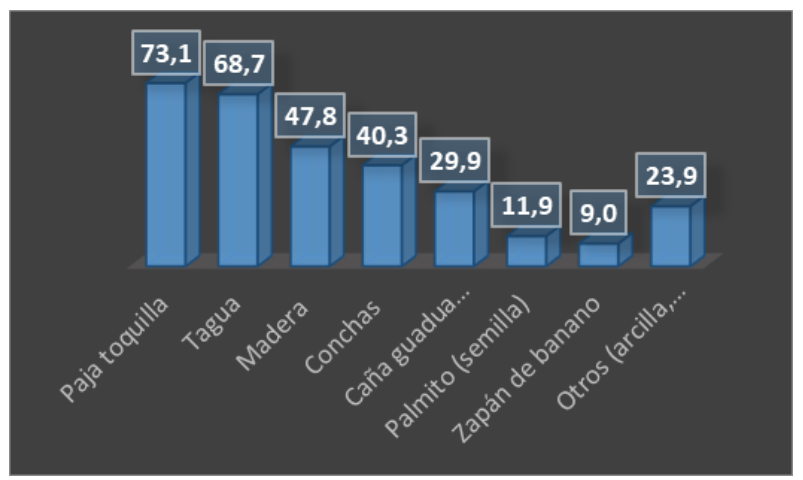

Las encuestas aplicadas a los turistas (85\% nacionales y $15 \%$ extranjeros) mostraron que el $75 \%$ compran de 1 a 3 objetos artesanales cuando visitan la provincia de Santa Elena, ver en el gráfico 2 el porcentaje de compra de productos según agrupación por materia prima. El $61 \%$ de ellos tiene un gasto promedio en artesanías entre 1 a 25 dólares, mientras que el 16\% invierte más de 100 dólares durante su estadía. En la muestra no existió una 
diferencia de gastos entre extranjeros y nacionales. Los encuestados señalaron además, en un $87 \%$, que la compra de artesanías es muy importante cuando visitan un lugar, indicando que entre las principales motivaciones está tener un recuerdo (42\%), adquirirlas por la estética que presentan (17\%) y tenerlas para obsequiarlas (14\%).

Gráfico 2: Preferencia de productos artesanales por parte de los visitantes.

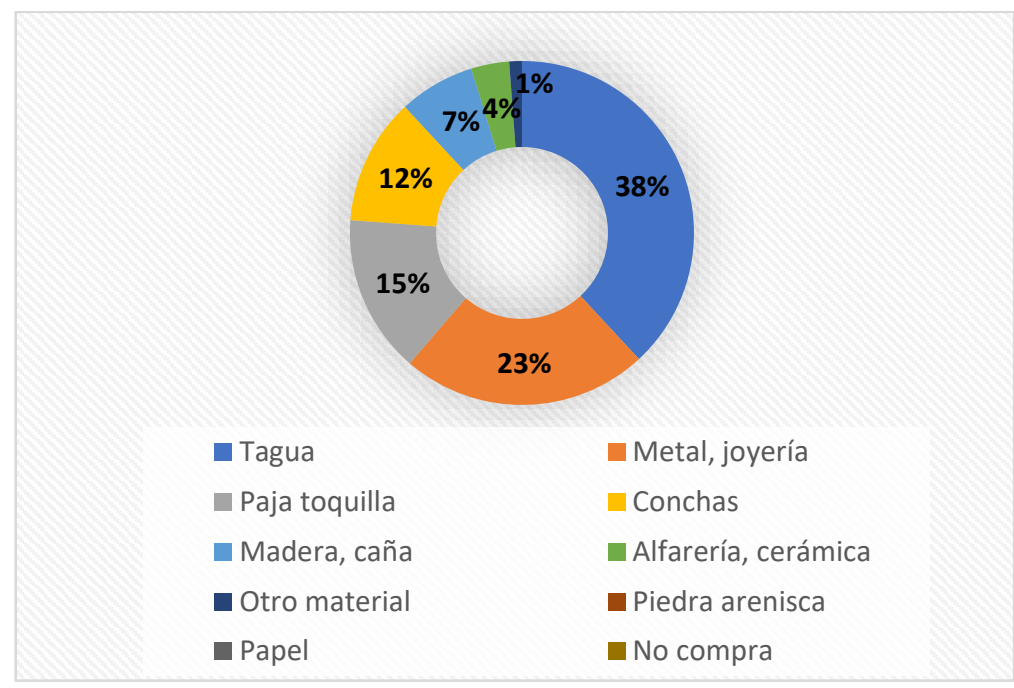

Los visitantes señalaron que tienen conocimientos sobre la producción artesanal en la zona gracias a las referencias generadas por amigos y familiares $(65,7 \%)$, la presencia de los productos en ferias y exposiciones $(64,2 \%)$, así como también por los viajes realizados anteriormente a la zona (52,2\%); el alcance de la publicidad de las artesanías fue reconocido por internet en un 34,3\%, mientras que la promoción realizada por las agencias de viajes, al igual que diarios y revistas tuvieron una respuesta del 17,9\% y $16,4 \%$ respectivamente. En estos datos se observa lo novedoso del alcance de la publicidad debido a las referencias que generan los visitantes que han llegado a este territorio, información que está por encima de la promoción que se realiza con el uso de la tecnología y de aquellas estrategias que aplican los Gobiernos Autónomos Descentralizados y los Ministerios encargados de este sector.

Una mayoría de los encuestados (77\%) señaló desconocer el significado de la artesanía comprada, y un porcentaje similar reconoce que las artesanías tendrían mayor valor simbólico, ancestral y económico si ellos tuviesen un conocimiento de lo que representan, así como su historia de producción. La importancia que representa para los visitantes la compra de productos artesanales es catalogada en un grado de relevancia alta (87\%).

\section{Discusión.}

Podemos destacar junto a Ciliane et al. (2014), que desde tiempos remotos las artesanías generan un impacto muy fuerte en la identidad de los territorios al momento de poner de manifiesto el arte que los ha caracterizado [14], este aspecto es lo que consideramos puede constituir un elemento esencial de valor y atracción para los visitantes, sin embargo, los resultados de la exploración muestran que prácticamente no es visible en las proyecciones de venta. 
De forma general los turistas compran las artesanías sin conocer su significado, este elemento puede retomar el valor que realmente tienen las artesanías autóctonas a través de una adecuada gestión y la identificación de un sistema de puesta en valor, a pesar de que los artesanos desconocen de estas posibilidades.

Esta realidad la vemos asociada al conjunto de la problemática del sector artesanal en Ecuador que en el caso de Santa Elena no se aleja mucho de la realidad del país, entre los aspectos más fácilmente detectables constan:

i) La falta de orientación al artesanado que ha provocado en muchos casos la explotación por parte de ONG's que hacen pedidos en cantidades, reconociéndoles valores mínimos.

ii) La escasa comercialización y el desconocimiento al respecto ha afectado la producción de artesanías en algunas de las familias que han terminado migrando a las ciudades cercanas.

iii)Cambios en los hábitos relacionados con la elaboración de los productos ofertados como consecuencia de la falta de un direccionamiento que fomente la revalorización y el rescate de lo tradicional del saber ancestral que aún mantienen las familias.

iv)Las exigencias de la demanda tienden a transformar y afectar la autenticidad de algunos elementos que disminuyen el simbolismo de lo ofertado.

De lo anterior se desprende la importancia de tener en cuenta esta problemática identificada, de la cual se deriva a su vez la no evidencia de un control hacia el uso adecuado de los recursos que forman parte de la materia prima de los artesanos (paja toquilla, tagua, caña), situación que arriesga su sostenibilidad, así como tampoco se pudo encontrar un registro detallado por parte de los entes reguladores del turismo y del sector artesanal sobre el consumo de las artesanías a nivel de la provincia que pueda auxiliar el análisis sobre los impactos que genera como un elemento dinamizador del desarrollo turístico. Los autores consideran que los resultados que se muestran pueden contribuir a suplir la falta de análisis sobre la contradictoria relación turismo y artesanía.

Si bien autores como Silva (2005) plantean que dentro del ámbito de los vínculos del turismo con el desarrollo de nuevas tecnologías y las artesanías, la mayor complejidad está en la rama de las artesanías autóctonas (3), consideramos que la viabilidad de esta relación está en el desarrollo de un turismo responsable y sostenible, a través de una sistemática información sobre el comportamiento de la demanda que, de acuerdo a este estudio, destaca el significado histórico-cultural y patrimonial como sostén esencial de la puesta en valor de las artesanías autóctonas y considerar que la perdida de este valor significa a su vez la pérdida o disminución de su demanda turística.

\section{Conclusiones}

> La provincia de Santa Elena cuenta con una producción artesanal autóctona variada con un potencial para captar la atención de los visitantes por la diversidad de productos y materia prima que utilizan en su confección generando ingresos que se complementan con otras actividades económicas que ejecutan. Estas artesanías han sobrevivido a las elaboradas de manera industrial gracias a la 
autenticidad plasmada en los saberes ancestrales de las hábiles manos de los artesanos que satisfacen a los visitantes con sus originales diseños.

$>$ Los resultados obtenidos reflejan condiciones propicias para la comercialización turística de las artesanías autóctonas que se confeccionan en la provincia, al tener en cuenta la información, identificación y análisis de los factores negativos que inciden en esta comercialización y contraponer a ello los valores históricos, patrimoniales y simbólicos que favorecen su puesta en valor turístico a través de una gestión sostenible.

$>$ Las investigaciones vinculadas a la producción artesanal, su significado como saber ancestral y los efectos que se generan en el territorio en estudio deben tener continuidad, considerando la falta de información y estudios al respecto que existe, incluso, a nivel nacional.

\section{Referencias Bibliográficas}

[1] Organización Mundial del Turismo - OMT. Panorama OMT del turismo internacional. Madrid, 2017. 16 p. ISBN 978-92-844-1904-3

[2] ECUADOR. Secretaría Nacional de Planificación y Desarrollo - SENPLADES. Plan Nacional para el Buen Vivir 2017-2021. Quito, 2017. 159 p.

[3] SILVA SALAZAR, Omar. Un modelo de comercialización de las artesanías ecuatorianas. Tesis (en opción al grado de Magíster en Seguridad y Desarrollo). Quito: Instituto de Altos Estudios Nacionales. 2005. 262 p.

[4] UNESCO. Índice Unesco Artesanía-Turismo. Informe elaborado por el Sr. Dominique Bouchart, Consultor- Sección de Artes, Artesanías y Diseño. División de Artes e Iniciativas Culturales. París, 2004. 46 p.

[5] Fernández de Paz, Esther. La Valorización Artesana y su Repercusión Turística. El Caso de Chile. PASOS. Revista de Turismo y Patrimonio Cultural, vol. 13, núm. 2, enero, 2015, pp. 375-393. Universidad de La Laguna. El Sauzal (Tenerife), España. Disponible en: http://www.redalyc.org/articulo.oa?id=88134125008 ISSN 1695-7121

[6] Tudorache Petronela. The importance of the intangible cultural heritage in the economy. En 3rd Global Conference on Business, Economics, Management and Tourism, 26-28 November 2015, Rome, Italy. Procedia Economics and Finance Volume 39 (2016) 731-736. Available online at https://www.sciencedirect.com/science/article/pii/S2212567116302714 ISSN $2212-5671$

[7] Turok Wallace, Marta. Análisis social de los artesanos y artesanas en Latinoamérica. Revista Artesanías de América N73. Centro Interamericano de Artesanías y Artes $\begin{array}{llll}\text { Populares. } & 2013 & \text { pp. } & 22-29\end{array}$ http://documentacion.cidap.gob.ec:8080/handle/cidap/1574 ISSN 0257-1625 
[8] Swanson, Kristen K., Timothy, Dallen J. Souvenirs: Icons of meaning, commercialization and commoditization, Tourism Management, Volume 33, Issue 3, June 2012, Pages 489-499. Disponible en http://www.sciencedirect.com/science/article/pii/SO26151771100207X

[9] Hernández Girón, José De la Paz, Yescas León, María, y Domínguez Hernández María Luisa. Factores de éxito en los negocios de artesanía en México. Revista Estudios gerenciales Vol. 23 No. 104 (Julio - Septiembre 2007) 77-99. Disponible en: https://www.sciencedirect.com/science/article/pii/S0123592307700189 ISSN 0123-5923

[10] ECUADOR. Ministerio de Turismo del Ecuador. Diseño del Plan Estratégico de Desarrollo de Turismo Sostenible para el Ecuador - PLANDETUR 2020. Quito, 2007. 536 p.

[11] Verónica Sión de Josse. Editorial en Revista País Productivo. Ministerio de Industria y Productividad del Ecuador. Diciembre 2012 - Enero 2013. 20 p. ISSN 13907522. Disponible en: https://www.industrias.gob.ec/wpcontent/uploads/downloads/2012/12/pais_productivo3.pdf

[12] PROECUADOR. Análisis del sector artesanías. Pro Ecuador Instituto de Promoción de Exportaciones e Inversiones. Marzo, 2013. 34 p. Disponible en http://www.proecuador.gob.ec/wpcontent/uploads/2013/11/PROEC_AS2013_A RTESANIAS.pdf Sin ISSN

[13] Fondo Nacional para el Fomento de las Artesanías - FONART (2015). Manual de diferenciación entre artesanía y manualidad. México. https://www.gob.mx/cms/uploads/attachment/file/107963/Manual_diferenciacio n_artesania_manualidad_2015.pdf

[14] Ciliane Ceretta, Caroline, Zamberlan dos Santos, Nara Rejane, Flores dos Santos Vilson. La valoración de las artesanías en el sur de Brasil a través del turismo. En Estudios y perspectivas en turismo Volumen 23 (2014) PP. 668 - 684. Disponible en http://www.scielo.org.ar/scielo.php?script=sci_arttext\&pid=S185117322014000400002 ISSN 1851-1732

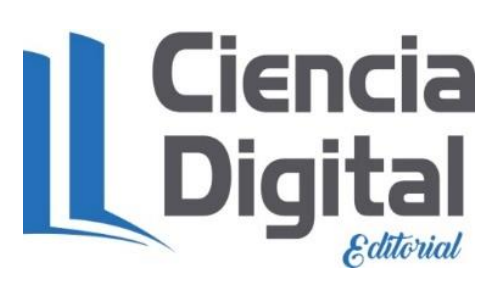




\section{Para citar el artículo indexado.}

Mendoza A., Ramírez C., Yumisaca J. \& Peralta S. (2019). Artesanías autóctonas como atractivo turístico en la provincia de Santa Elena, Ecuador. Revista electrónica Explorador Digital 3(2), 5-15. Recuperado desde:

http://cienciadigital.org/revistacienciadigital2/index.php/exploradordigital/article/view/415/9 $\underline{44}$

\section{Ciencia \\ Digital \\ Editorial}

El artículo que se publica es de exclusiva responsabilidad de los autores y no necesariamente reflejan el pensamiento de la Revista Explorador Digital.

El articulo queda en propiedad de la revista y, por tanto, su publicación parcial y/o total en otro medio tiene que ser autorizado por el director o editor de la Revista Explorador Digital.
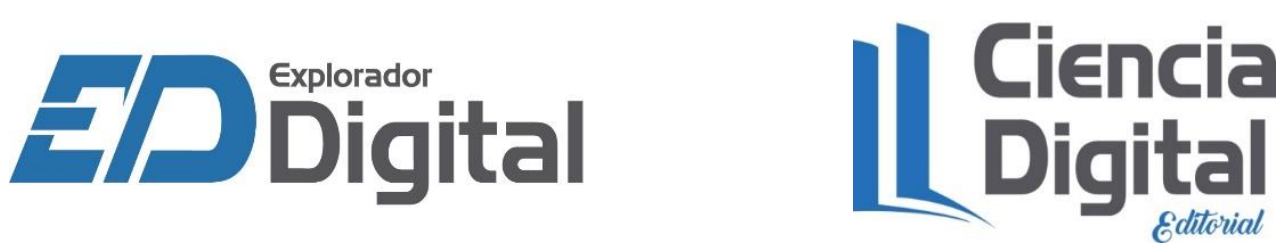